\title{
Transcription-frequency-dependent modulation of an attenuator in a ribosomal protein-RNA polymerase operon requires an upstream site
}

\author{
Keith L. Steward, $\uparrow$ Rebecca St Pierre and Thomas Linn \\ Author for correspondence: Thomas Linn. Tel: +1 5196613426 . Fax : +1 5196613499 . \\ e-mail: tlinn@julian.uwo.ca
}

Department of

Microbiology and

Immunology, Faculty of

Medicine, University of

Western Ontario, London,

Ontario, Canada N6A 5C1

\begin{abstract}
Although the attenuator located between the ribosomal protein and RNA polymerase gene domains of the Escherichia coli rp/KAJLrpoBC operon has a maximum termination efficiency of $80 \%$, the level of termination is diminished with decreasing transcription frequency. In this report, the use of transcriptional fusions to further investigate the mechanism of transcriptionfrequency-dependent regulation is described. The termination efficiency of two other weak terminators was assayed over a wide range of transcription frequencies programmed by different strength promoters. The results indicated that a decrease in termination efficiency with decreasing transcription frequency is not an inherent property of weak terminators. Deletion of the 165 bp segment located 439-274 bp upstream of the attenuator abrogated the difference in termination efficiency normally seen between high and low levels of transcription. This suggests that a cis-acting site located in this upstream region is necessary for transcription-frequency-dependent modulation of the attenuator's function. However, this site apparently works only in combination with the attenuator, since it did not cause transcriptionfrequency-dependent modulation when placed upstream of two other weak terminators. Analysis of the readthrough frequencies of single or tandem copies of the attenuator indicated that the transcription complexes which pass through the attenuator have not been converted to termination-resistant complexes in a manner analogous to the $\mathrm{N}$-mediated antitermination system of lambda. Finally, an examination of termination efficiency in three nusA mutants suggested that although NusA increases readthrough at the attenuator it is not directly involved in transcription-frequency-dependent modulation.
\end{abstract}

Keywords : transcription termination, attenuator, RNA polymerase, Escherichia coli

\section{INTRODUCTION}

The synthesis of RNA polymerase in Escherichia coli is not constitutive, but is autogenously regulated and correlated with the growth requirements of the cell. The minimal core RNA polymerase $\left(\beta \beta^{\prime} \alpha_{2}\right)$, which is competent for elongating RNA transcripts, can associate with one of at least six sigma subunits during transcription initiation (Ishihama, 1993). Consistent with this transient interaction, the sigma factors are present

\footnotetext{
† Present address: Sequana Therapeutics Inc., 11099 North Torrey Pines Road, La Jolla, CA 92037, USA.
}

in the cell at lower levels relative to core enzyme. The primary sigma factor utilized during exponential growth, $\sigma^{70}$, is present at approximately $40 \%$ of the level of core enzyme (Iwakura et al., 1974; Engbaek et al., 1976; Hayward \& Fyfe, 1978; Kawakami et al., 1979). The $\alpha$ subunit however is synthesized in excess of that required to assemble core enzyme (Iwakura et al., 1974; Engbaek et al., 1976; Pedersen et al., 1976; Hayward \& Fyfe, 1978; Kawakami et al., 1979). Therefore, the assembly of core enzyme, and thus the global transcription capacity of the cell, appears to be limited by the synthesis of the $\beta$ and $\beta^{\prime}$ subunits.

The $\beta$ and $\beta^{\prime}$ subunits are encoded by the $r p o B$ and $r p o C$ genes, respectively, which are located at the distal end of 


\section{Gene}

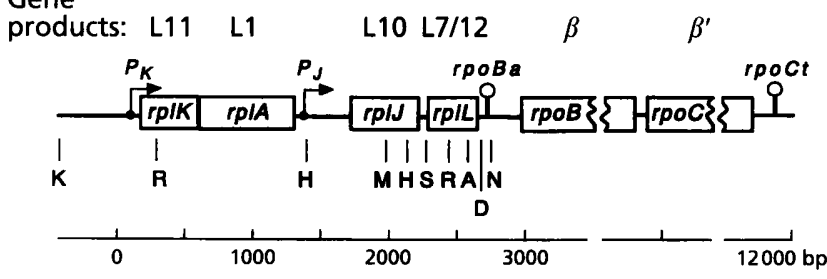

Fig. 1. Organization of the rp/KAJLrpoBC ribosomal protein and RNA polymerase gene cluster. The rp/KAIL region is drawn to scale. The $r p / K p\left(P_{K}\right)$ and $r p / J p\left(P_{j}\right)$ promoters and the direction of transcription are indicated by the bent arrows. Hairpins indicate the locations of the rpoBa attenuator and rpoCt terminator. The positions of restriction sites used in the construction of the rplrpo-lacZ fusions are drawn below the map. A, Alul (nt 2623); D, Ddel (2672); H, Hindlil (2154 and engineered at 1339); K, Kpnl (-428); M, Smal (1986); N, Narl (2730); R, EcoRI (280 and 2444); and S, Swal (2279). The nucleotide numbering is according to Post et al. (1979).

the $12 \mathrm{~kb}$ rplKAJLrpoBC gene cluster (Fig. 1). The four upstream genes encode ribosomal proteins and all six genes are cotranscribed in a complex pattern (Steward $\&$ Linn, 1991). Promoters of approximately equal strength are located upstream of $r p l K(r p l K p)$ and $r p l J$ $(r p l J p)$ (Fig. 1), and since there is no terminator between them, both promoters are responsible for transcription of the rplJLrpoBC genes. However, during exponential growth on rich media, the $r p o B C$ genes are transcribed at about one-fifth the frequency of the upstream $r p l J L$ genes due to termination at an attenuator $(r p o B a)$ located within the $r p l L-r p o B$ intercistronic region.

A number of studies have indicated that there are both transcriptional and translational control mechanisms involved in determining the intracellular level of RNA polymerase (Steward \& Linn, 1992; Dykxhoorn et al., 1996; and references cited therein). Recently, we showed that the translation of $r p o B$ and $r p o C$ mRNA is reduced in response to increased concentrations of either $\beta$ or $\beta^{\prime}$ (Dykxhoorn et al., 1996). However, when the concentration of all the holoenzyme subunits is increased, the transcription of $r p o B C$ is reduced, primarily by lowering initiation at $r p l K p$ and $r p l J p$ (Dykxhoorn et al., 1996). These results suggest that excess production of the $\beta$ and $\beta^{\prime}$ subunits can autogenously regulate $r p o B C$ expression at either the transcriptional or translational level depending on whether they are assembled into holoenzyme or remain as individual polypeptides.

Another aspect of autogenous control of RNA polymerase synthesis may involve regulation of termination at the $r p o B a$ attenuator. The $r p o B a$ sequence resembles a simple terminator in that it produces an RNA transcript which can fold into a 11 bp GC-rich hairpin followed by five consecutive $U$ residues. However, we have shown previously that readthrough of $r p o B a$ increases from a minimum of $20 \%$ to greater than $60 \%$ as the frequency of transcription of the region containing rpoBa decreases (Steward \& Linn, 1992). The finding that the frequency of termination at $r p o B a$ is inversely modulated by transcription frequency suggests an autogenous control mechanism that senses the level of functional polymerase, and in particular the level of transcription of rplKAJLrpoBC. Accordingly, as transcription into $r p o B a$ is reduced, there is a partial compensatory response by increasing readthrough of $r p o B a$ and thus transcription of the $r p o B C$ genes.

In the current study we have further examined the mechanism of the transcription-frequency-dependent modulation of $r p o B a$ termination efficiency. Previously, we observed that the level of transcription of $r p o B$ relative to the upstream $r p l J L$ genes was decreased in a nusA1 mutant strain (Ralling \& Linn, 1987). Subsequently, we found that the addition of either NusA or NusG to a purified in vitro transcription system increased the readthrough of $r p o B a$ (Linn \& Greenblatt, 1992). Since these results suggested NusA and NusG can affect termination of $r p o B a$, and because they were first identified as components of the N-mediated antitermination system in phage $\lambda$ (Friedman, 1988; Das, 1992; Greenblatt $e t$ al., 1993), we examined whether aspects of the mechanism operating at $r p o B a$ were analogous to those in $\lambda$. We found that, analogous to the $\lambda$ system, modulation of termination at $r p o B a$ appears to require upstream sequences. However, in contrast to $\lambda$, this upstream region does not function with other terminators and the polymerase molecules that read through $r p o B a$ have not been converted into stable terminationresistant complexes.

\section{METHODS}

E. coli strains. MG2 is a $\Delta(\operatorname{argF}-\mathrm{lac}) U 169$ derivative of MG1655, MG4 is a recA56 derivative of MG2 (Ralling et al., 1985). C600 was described by Appleyard (1954). DH5 $\alpha$

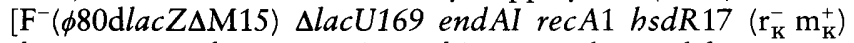
deoR supE44 thi-1 $\lambda^{-}$gyrA96 relA1] was obtained from BRL. MG1457 [nusA1 argG ::Tn5 $\Delta(\operatorname{argF-lac})$ U169] was constructed by $\mathrm{P} 1$ cotransduction of the kanamycin resistance and nusA1 markers from K1457 to MG2. MG1914 [nusA10(Cs) $\arg G:: \operatorname{Tn} 5 \Delta(\arg F-l a c) U 169$ ] was constructed by P1 cotransduction of the kanamycin resistance and nusA10(Cs) markers from K1914 to MG2. MG3903 [nusA11(Ts) Tn10 $\Delta(\arg F-l a c) U 169]$ was constructed by $\mathrm{P} 1$ cotransduction of the tetracycline resistance and $n u s A 11$ (Ts) markers from K3909 to MG2. K1457, K1914 and K3903 were kindly supplied by D. Friedman (University of Michigan).

Recombinant DNA. Transcriptional or operon fusions were constructed in the lambda TL61 transcriptional fusion vector (Linn \& St Pierre, 1990). Restriction endonucleases, DNA modifying enzymes and linkers were obtained from Boehringer Mannheim, Pharmacia or New England Biolabs. Insert DNA fragments and vector phage arms were produced by restriction endonuclease digestion of source DNA and resolved by agarose gel electrophoresis. Appropriate bands were excised from the standard agarose or Nusieve GTG (FMC BioProducts) agarose gels and purified by the GeneClean process (Bio101) with final resuspension of the DNA in TE buffer $(10 \mathrm{mM}$ Tris/HCl, $1 \mathrm{mM}$ EDTA, pH 7.5). When required, staggered ends were filled in using the Klenow enzyme. Recombinant phage were recovered from ligation 
Table 1. Details of rplrpo-lacZ transcriptional fusions

\begin{tabular}{|c|c|c|c|c|}
\hline Fusion & Features* & $5^{\prime}$ end $\dagger$ & $3^{\prime}$ end $\dagger$ & Source \\
\hline KS166 & Pant244-rplJLrpoBa & HindIII (1339) & NarI $(2730)$ & $\begin{array}{l}\text { Steward \& Linn } \\
\text { (1992) }\end{array}$ \\
\hline KS169 & Pant285-rplJL & HindIII (1339) & DdeI (2672) & $\begin{array}{l}\text { Steward \& Linn } \\
\text { (1992) }\end{array}$ \\
\hline KS170 & Pant285-rplJLrpoBa & HindIII (1339) & Narl (2730) & $\begin{array}{l}\text { Steward \& Linn } \\
\text { (1992) }\end{array}$ \\
\hline KS195 & Pant224-rplJL & HindIII (1339) & DdeI (2672) & $\begin{array}{l}\text { Steward \& Linn } \\
\text { (1992) }\end{array}$ \\
\hline KS253 & Pant285-rplL & SwaI (2279) & DdeI (2672) & This study \\
\hline KS257 & Pant285-rplLrpoBa & SwaI (2279) & $\operatorname{NarI}(2730)$ & This study \\
\hline KS264 & Pant285-rpl'L & EcoRI (2444) & $D d e \mathrm{I}(2672)$ & This study \\
\hline KS265 & Pant285-rpl'LrpoBa & EcoRI (2444) & $\operatorname{NarI}(2730)$ & This study \\
\hline KS266 & Pant1045-rplJLrpoBa & HindIII (1339) & Narl (2730) & This study \\
\hline KS267 & Pant1045-rplJL & HindIII (1339) & $D d e I(2672)$ & This study \\
\hline KS269 & $\begin{array}{l}\text { Pant224-rplJLrpoBa- } \\
\quad r p o B a\end{array}$ & HindIII (1339) & $\begin{array}{l}\text { NarI }(2730)+D d e \mathrm{I} \\
(2672)-N a r I(2730)\end{array}$ & This study \\
\hline KS270 & Pant1045-rpl'L & EcoRI (2444) & $\operatorname{DdeI}(2672)$ & This study \\
\hline KS271 & Pant1045-rpl'LrpoBa & EcoRI $(2444)$ & NarI (2730) & This study \\
\hline KS285 & Pant1045-rplL & SwaI (2279) & DdeI (2672) & This study \\
\hline KS286 & Pant1045-rplLrpoBa & SwaI (2279) & NarI (2730) & This study \\
\hline KS287 & $\begin{array}{l}\text { Pant285-rplJLrpoBa- } \\
\quad r p o B a\end{array}$ & HindIII (1339) & $\begin{array}{l}\text { NarI }(2730)+D d e \mathrm{I} \\
(2672)-N a r I(2730)\end{array}$ & This study \\
\hline KS305 & Pant285-rpl'LrpoBa & AluI (2623) & $\operatorname{Narl}(2730)$ & This study \\
\hline KS311 & Pant285-rpl'L & AluI $(2623)$ & DdeI (2672) & This study \\
\hline KS312 & Pant1045-rpl'L & $A l u \mathrm{I}(2623)$ & DdeI (2672) & This study \\
\hline KS313 & Pant1045-rpl'LrpoBa & AluI (2623) & NarI (2730) & This study \\
\hline
\end{tabular}

* Primes before a gene designation indicate truncation of the $5^{\prime}$ end of the gene; primes after a gene designation indicate truncation of the $3^{\prime}$ end of the gene.

$\dagger$ The restriction sites used to generate the $5^{\prime}$ and $3^{\prime}$ endpoints of the rplrpo region are listed. Restriction site coordinates (in parentheses) are according to the numbering of Post et al. (1979).

reactions by packaging in vitro and plating the reactions on fresh lawns of $\mathrm{C} 600$ as previously described (Maniatis et al., 1982). Recombinant plasmids were recovered from ligation reactions by transformation of frozen competent DH5 $\alpha$ cells prepared as described by Hanahan (1985). All phage and plasmid constructs were screened by extensive restriction endonuclease digestion analyses. Critical regions of some constructs were confirmed by dideoxy sequencing. Phage DNA was prepared from plate lysates by the small-scale method (Sambrook et al., 1989) or a modification of the $\mathrm{ZnCl}_{2}$ method (Santos, 1991). Plasmid DNA for screening was prepared by a modification of the method of Holmes $\&$ Quigley (1981). Preparative amounts of plasmid were purified by the Qiagen method.

Transcriptional fusions. Transcriptional fusions of rplrpo regions to lac $Z$ were constructed in the lambda TL61 fusion vector (Linn \& St Pierre, 1990). Details of genetic and regulatory features contained in the various fusions are presented in Table 1. Fusions KS166, KS169, KS170, KS176 and KS195 were described previously (Steward \& Linn, 1992).

(i) Source of promoters for driving transcription of rp/rpo segments and $\mathbf{t} \mathbf{2}$ terminators. The original recombinant phage constructs containing the tac (TAC1), Pant285 ( $\mathrm{Pa} 285)$, Pant224 (KS200) and Pant219 (Pa219) promoters were described previously (Steward \& Linn, 1992). The additional constructs used in this study containing the Pant222 and Pant1045 promoters were made in the same way as the Pantcontaining phage listed above (Steward \& Linn, 1992).

(ii) Fusions containing $t R 2^{2}$ and $t R 2^{9}$ downstream of different promoters. In a series of constructs, the $t R 2^{2}$ and $t R 2^{9}$ terminators isolated as $440 \mathrm{bp} S m a \mathrm{I}-\mathrm{XbaI}$ fragments from pKL600tR2 ${ }^{2}$ and pKL600tR2 ${ }^{9}$ (Cheng et al., 1991) were inserted between various promoters and the lac $Z$ gene. A summary of the features contained in the fusions is presented in Table 2. tR 35 and tR29 were constructed by inserting the $t R 2^{2}$ - and $t R 2^{9}$-containing fragments between the XbaIgenerated left arm of TL61 and the Sacl-generated right arm of KS211. KS211 was constructed from the EcoRI-generated left arm of TL61 and EcoRI-generated right arm of Pa285. This construction places a $\mathrm{SacI}$ site downstream of the EcoRI site in Pa285. tR33 and tR34 were constructed from the EcoRIgenerated left arm of tR 35 and the EcoRI-generated right arm of $\mathrm{Pa} 222$ or Pa1045, respectively. tR44, tR47, tR48 and tR49 were constructed from the EcoRI-generated left arm of tR29 and the EcoRI-generated right arm of $\mathrm{Pa} 1045, \mathrm{~Pa} 222, \mathrm{~Pa} 219$ or TAC1, respectively.

(iii) Fusions used in deletion analysis to delineate an upstream modulation site. For each of the Pant285 and Pant1045 promoters, fusions were constructed with or without the 58 bp rpoBa-containing $D d e \mathrm{I}-\mathrm{NarI}$ region such that they 
contained the $r p l$ DNA extending 1375 bp (KS169 and KS170, and KS267 and KS266, respectively), $439 \mathrm{bp}$ (KS253 and KS257, and KS285 and KS286, respectively), 274 bp (KS264 and KS265, and KS270 and KS271, respectively) or $95 \mathrm{bp}$ (KS311 and KS305, and KS312 and KS313, respectively) upstream of the $r p o B a$ termination site. The $3^{\prime}$ end of the $r p l$ segment in those fusions that lacked the attenuator was at the Ddel site at nt 2672 (nucleotide numbering according to Post et al., 1979), while the $3^{\prime}$ end in those fusions that contained $r p o B a$ was at the $\mathrm{NarI}$ site at nt 2729. The $5^{\prime}$ ends of the $r p l$ segments were provided by the HindIII (engineered at nt 1339), SwaI (2279), EcoRI (2444) or AluI (2623) sites (Table 1). KS169 and KS170 were described previously (Steward \& Linn, 1992). KS267 was constructed from the XhoI-generated left arm of KS169 and the XhoI-generated right arm of Pa1045. KS266 was constructed from the Xhol-generated left arm of KS170 and the XhoI-generated right arm of Pa1045. KS253 was constructed from the SwaI-generated left arm of KS167, 1692 bp SmaI-BamHI fragment of KS203 (which is identical to the SmaI-BamHI fragment of $\mathrm{Pa} 285$ ) and BamHI-generated right arm of TL61. KS257 was constructed from the SwaIgenerated left arm of KS170, 1692 bp SmaI-BamHI fragment of Pa285 and BamHI-generated right arm of TL61. KS285 was constructed from the SwaI-generated left arm of KS169, $1692 \mathrm{bp} \mathrm{SmaI-BamHI}$ fragment of $\mathrm{Pa} 1045$ and BamHIgenerated left arm of TL61. KS286 was constructed from the SwaI-generated left arm of KS170, 1692 bp SmaI-BamHI fragment of $\mathrm{Pa} 1045$ and BamHI-generated fragment of TL61. KS264 and KS265 were both constructed with the EcoRIgenerated right arm of $\mathrm{Pa} 285$, and the EcoRI-generated left arm of KS167 or KS170, respectively. KS270 and KS271 were both constructed with the EcoRI-generated right arm of $\mathrm{Pa} 1045$, and the EcoRI-generated right arm of KS169 or KS170, respectively. KS311 was constructed from the XbaIgenerated right arm of TL61, 97 bp Xbal-XhoI fragment of pKS309 and XhoI-generated right arm of Pa285. pKS309 contains the $60 \mathrm{bp}$ XhoI-DdeI (blunted) fragment of pKS290 inserted between the XhoI and SmaI sites of pTL61T (Linn \& St Pierre, 1990). pKS290 contains the $121 \mathrm{bp}$ AluI (blunted)-HindIII fragment of pKS284 inserted between the SmaI and HindIII sites of pTL61T. pKS284 contains the 303 bp EcoRI-HindIII fragment of KS265 that contains $r p o B a$, inserted between the EcoRI and HindIII sites of pGEM-11Zf(). KS305 was constructed from the $X b$ aI-generated left arm of TL61, 154 bp Xbal-XhoI fragment of pKS290 and XhoIgenerated right arm of $\mathrm{Pa} 285$. KS312 was constructed from the XbaI-generated left arm of TL61, $97 \mathrm{bp} \mathrm{XbaI-XhoI} \mathrm{fragment}$ of pKS309 and Xhol-generated right arm of Pa1045. KS313 was constructed from the EcoRI-generated left arm of KS305 and EcoRI-generated right arm of Pa1045.

(iv) Fusions used to test readthrough of tandem copies of rpoBa. These fusions contained either Pant285 (KS169, KS170, KS287) or Pant224 (KS195, KS166, KS269) fused upstream of an rplrpo segment extending from the engineered HindIII site at nt 1339 to the $D d e I$ site located $43 \mathrm{bp}$ before the $r p o B a$ termination site, and contained in addition no (KS169, KS195), one (KS170, KS166), or two (KS287, KS269) tandem copies of the DdeI-NarI rpoBa-containing region (Table 1). KS166, KS169, KS170 and KS195 were described previously (Steward \& Linn, 1992). KS287 was constructed from the XhoIgenerated left arm of KS269 and Xhol-generated right arm of $\mathrm{Pa} 285$. KS269 was constructed from the XhoI-generated left arm of KS176, 480 bp Sal I-SwaI fragment of KS170 and SwaIgenerated right arm of KS166.

(v) Fusions containing the 393 bp rp/L fragment. A summary of the features contained in the fusions is presented in Table 3.
tR46 was constructed from the $X b$ aI-generated right arm of KS285 and Xbal-generated left arm of TL61. tR40 and tR43 were constructed from the Smal-generated left arm of tR35 and BamHI-generated right arm of $\mathrm{Pa} 222$ surrounding, respectively, the SalI(blunted)-Bam HI Pant1045-containing fragment of KS285 or the SalI(blunted)-BamHI Pant285containing fragment of KS253. tR45 was constructed from the Smal-generated left arm of tR29, BamHI-generated right arm of $\mathrm{Pa} 222$ and $\mathrm{Sal}$ (blunted)-BamHI Pant1045-containing fragment of KS285.

Lysogenization and $\beta$-galactosidase assays. Monolysogens of each recombinant lac $Z$ transcriptional fusion phage were recovered in MG4 as described previously (Linn \& St Pierre, 1990) with the exception of the lysogens described in Table 4. Lysogens were confirmed as monolysogens using the TER excision test (Linn \& St Pierre, 1990; Mousset \& Thomas, 1969). $\beta$-Galactosidase assays were performed essentially as described by Miller (1972), except that cultures were grown in AB medium (Clark \& Maaloe, 1967) supplemented with thiamin $\left(2 \mathrm{mg} \mathrm{ml}^{-1}\right), 0.4 \%(\mathrm{w} / \mathrm{v})$ glucose and $0.4 \%(\mathrm{w} / \mathrm{v})$ Casamino acids. Samples of the culture were taken at an optical density of 15 Klett units (Klett-Summerson colorimeter), then $30-500 \mu \mathrm{l}$ cells were permeabilized by the addition of $20 \mu \mathrm{l}$ chloroform and $10 \mu \mathrm{l} 0 \cdot 1 \%$ (w/v) SDS in a final volume of $800 \mu \mathrm{l}$. All $\beta$-galactosidase values reported in the tables and figures represent the means of six to ten assays performed on three or more independent cultures and had standard deviations that ranged from 1 to $5 \%$.

\section{RESULTS \\ Weak terminators do not exhibit modulation of termination}

Previously, we showed that the attenuator preceding the $r p o B$ gene $(r p o B a)$ is not fixed in its termination efficiency, but is modulated by the frequency with which transcription complexes enter the attenuator (Steward \& Linn, 1992). In contrast, two other well-characterized terminators, rrnBT2 (Brosius et al., 1981) and $\lambda t R 2$ (Schmidt \& Chamberlin, 1987), were found to meet the expectation of constant termination efficiency independent of transcription frequency (Steward \& Linn, 1992). One concern, however, was that these two terminators are very efficient, with termination frequencies of greater than $95 \%$, whilst the maximum frequency of termination at $r p o B a$ only approaches $80 \%$. Perhaps termination efficiency at weaker terminators would be modulated by transcription frequency in the same manner as $r p o B a$. To test this directly we examined termination at two mutated versions of $\lambda t R 2$. The $t R 2^{2}$ terminator has a CG base pair in the stem replaced by a GC base pair, while the $t R 2^{9}$ terminator has two adjacent GC base pairs replaced by two CG base pairs with an additional AU base pair added to the stem (Cheng et al., 1991). Although the stability of the stem in these variants should be as great $\left(t R 2^{2}\right)$ or greater $\left(t R 2^{9}\right)$ than wild-type $t R 2$, both have reduced termination efficiency. We tested their response to varying transcription frequency by constructing a series of transcrip- 
Table 2. Termination efficiency of weak terminators as transcription frequency is varied

\begin{tabular}{|c|c|c|c|c|c|c|c|c|}
\hline \multirow[b]{2}{*}{ Promoter } & \multicolumn{2}{|c|}{ Promoter alone } & \multicolumn{3}{|c|}{$+t R 2^{2}$} & \multicolumn{3}{|c|}{$+t R 2^{g}$} \\
\hline & Fusion & $\beta$-Gal (units) ${ }^{*}$ & Fusion & $\beta$-Gal (units) ${ }^{*}$ & Term $(\%) \dagger$ & Fusion & $\beta$-Gal (units) ${ }^{*}$ & Term $(\%) \dagger$ \\
\hline Ptac & TAC1 & 13588 & - & - & - & $\mathrm{tR} 49$ & 4333 & 68 \\
\hline Pant 285 & $\mathrm{~Pa} 285$ & 7199 & $\mathrm{tR} 35$ & 1436 & 80 & tR29 & 2329 & 68 \\
\hline Pant219 & $\operatorname{Pa} 219$ & 2891 & - & - & - & tR48 & 952 & 67 \\
\hline Pant222 & $\mathrm{Pa} 222$ & 1260 & tR33 & 128 & 90 & $\mathrm{tR} 47$ & 429 & 66 \\
\hline Pant1045 & $\mathrm{Pa} 1045$ & 511 & tR34 & 52 & 90 & $\mathrm{tR} 44$ & 161 & 68 \\
\hline
\end{tabular}

* $\beta$-Galactosidase activities ( $\beta$-gal) were determined as described in Methods.

+ Percentage termination frequencies (term) were calculated as $100 \times[1-(\beta$-gal units from the fusion containing both the promoter and terminator $\div \beta$-gal units from the fusion containing only the promoter) $]$.

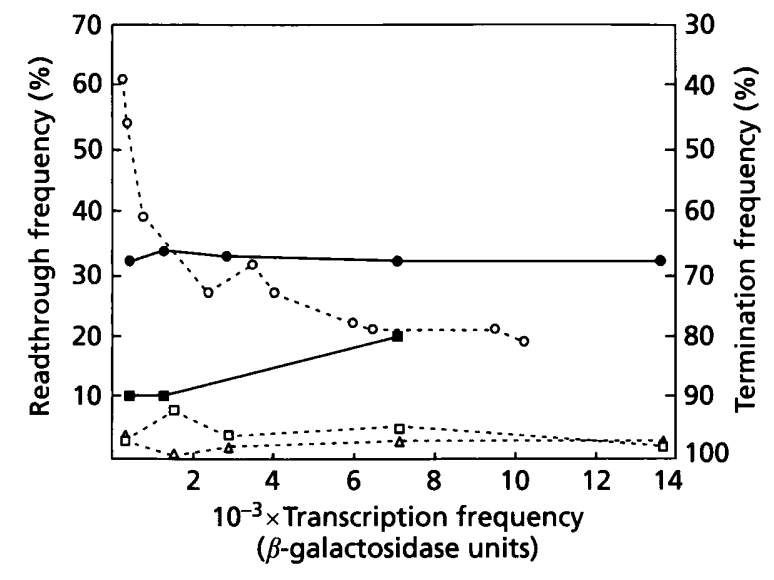

Fig. 2. Termination efficiency of $t R 2^{2}$ and $t R 2^{9}$ as a function of transcription frequency. The frequency of transcription entering the terminators is plotted versus the readthrough or termination frequencies presented in Table 2. The curve for $t R 2^{2}$ is indicated by filled squares and for $t R 2^{9}$ by filled circles. For comparison, the previously determined curves (Steward \& Linn, 1992) for rpoBa (open circles), tR2 (open squares) and rrnBT2 (open triangles) are also shown.

tional fusions in which each terminator, carried on a $440 \mathrm{bp}$ fragment, was inserted between different strength promoters and the lac $Z$ gene. The promoters used included Ptac and four variants of the P22 ant promoter. The Pant variants differ from one another by single bp changes in the -10 and -35 regions (Moyle et al., 1991). Although promoter strength is affected, all Pant variants initiate at the same position and produce identical RNA transcripts. These fusions were assembled on $\lambda$ vectors so a single copy could be stably integrated into the bacterial chromosome (see Methods). The termination efficiencies were then calculated from the $\beta$-galactosidase levels produced in lysogens containing fusions with or without the terminator. As seen in Table 2 and Fig. 2, the frequency of readthrough of $t R 2^{2}$ or $t R 2^{9}$ did not increase with decreasing transcription frequency. The weakest terminator, $t R 2^{9}$, showed a constant termination efficiency of approximately $68 \%$ as transcription frequency was varied over a 27 -fold span, indicating that at least these weaker terminators do not show transcription-frequency-dependent modulation.

\section{Identification of an upstream region required for modulation of rpoBa efficiency}

If the termination decision at $r p o B a$ was regulated by a mechanism similar to the $\mathrm{N}$-mediated antitermination system of $\lambda$, then one might predict that sequences located upstream of $r p o B a$ may be required for modulation of $r p o B a$ efficiency. To test this model decreasing amounts of DNA upstream of $r p o B a$ were used to construct two sets of fusions, with and without $r p o B a$ fused upstream of $l a c Z$. In those constructs lacking $r p o B a$, the $r p l J L$ sequences present in the fusion ended at the DdeI site 43 bp before the rpoBa termination site. The set of fusions that contained $r p o B a$ extended to the NarI site $15 \mathrm{bp}$ beyond the termination site. $\beta$-Galactosidase activity measurements for the first set of fusions revealed the transcription frequencies just prior to the attenuator, while measurements for the second set revealed readthrough frequencies. The transcriptionfrequency-dependent modulation was tested by driving transcription of the constructs with either the strong Pant 285 or weak Pant1045 promoters. These fusions were assembled on lambda vectors and the $\beta$-galactosidase levels were measured in monolysogens of the recombinant fusion phage as described in Methods. As observed earlier (Steward \& Linn, 1992), fusions containing virtually the complete upstream region extending from the normal position of $r p l J p$, but which had this endogenous promoter replaced, showed an approximately twofold higher readthrough frequency at $r p o B a$ when transcription was driven by the weak Pant 1045 promoter ( $42 \%$ readthrough) as compared to the strong Pant 285 (23\%) (Fig. 3). A similar difference in readthrough frequency was observed when the DNA upstream of $r p o B a$ was shortened to $439 \mathrm{bp}$. However, when the region was reduced to 274 bp the difference between low $(25 \%)$ and high $(24 \%)$ frequency tran- 


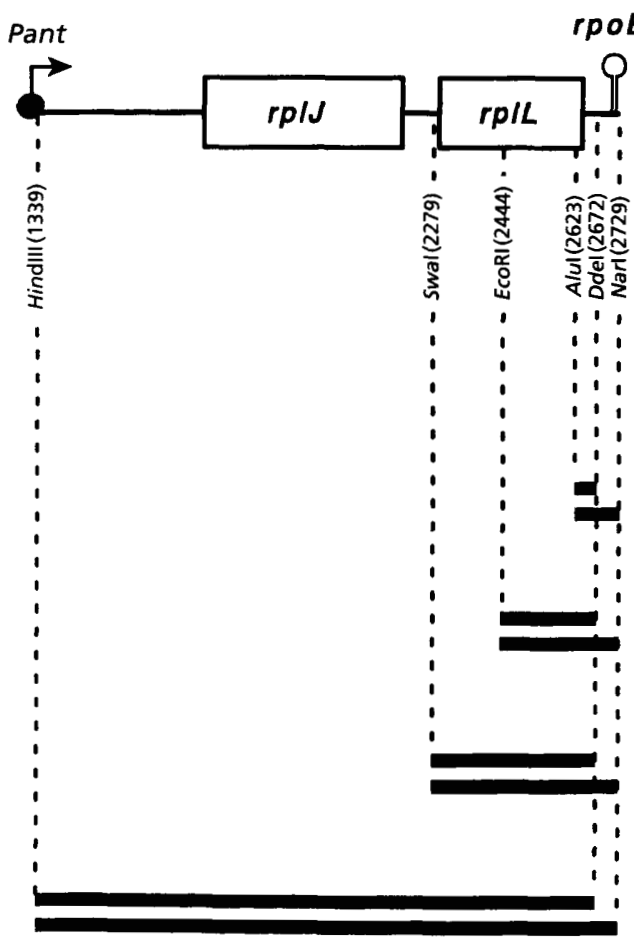

\begin{tabular}{|c|c|c|c|c|c|c|}
\hline \multirow{3}{*}{$\begin{array}{c}\text { Size of } \\
\text { upstream } \\
\text { region (bp) }\end{array}$} & \multicolumn{3}{|c|}{ Pant285 } & \multicolumn{3}{|c|}{ Pant1045 } \\
\hline & \multicolumn{2}{|c|}{$\beta$-Gal (units) } & \multirow{2}{*}{ RT (\%) } & \multicolumn{2}{|c|}{$\beta$-Gal (units) } & \multirow[t]{2}{*}{ RT (\% } \\
\hline & $\begin{array}{c}\text {-rpoBa } \\
\text { (Ddel) }\end{array}$ & $\begin{array}{c}+r p o B a \\
(\text { Narl) }\end{array}$ & & $\begin{array}{l}\text {-rpoBa } \\
\text { (Ddel) }\end{array}$ & $\begin{array}{c}+ \text { rpoBa } \\
\text { (Narl) }\end{array}$ & \\
\hline 95 & 5694 & 1815 & 32 & 371 & 102 & 27 \\
\hline 274 & 5754 & 1392 & 24 & 443 & 112 & 25 \\
\hline 439 & 6134 & 1502 & 24 & 402 & 165 & 41 \\
\hline 1379 & 6388 & 1458 & 21 & 427 & 181 & 42 \\
\hline
\end{tabular}

Fig. 3. Deletion analysis of the upstream region required for the transcription-frequency-dependent modulation of $r p o B a$ termination. lac $Z$ transcriptional fusions were constructed with and without the rpoBa attenuator and varying amounts of proximal DNA (filled bars), each with either the strong Pant285 or the weak Pant1045 promoters. For each fusion, the size of the region upstream of the rpoBa termination site, the $\beta$-galactosidase activity measurements $\left(\beta\right.$-gal) for $3^{\prime}$ fusion sites located $43 \mathrm{bp}$ before (Ddel) and $15 \mathrm{bp}$ beyond (Narl) rpoBa, and the percentage readthrough (RT) are shown. Restriction sites used as endpoints for the fusions are indicated.

scription disappeared. A further deletion, leaving only $95 \mathrm{bp}$ upstream of $r p o B a$ also showed no greater readthrough when transcription was driven by a weak promoter, but the mean of the readthrough levels at high and low transcription frequency increased somewhat to $29.5 \%$. This higher level of readthrough may be due to the closer proximity of the Pant sequences to $r p o B a$, which partially inhibits function of the terminator. These results suggest that a cis-acting site located in the $165 \mathrm{bp}$ Swal-EcoRI interval is required for $r p o B a$ modulation.

\section{Modulation of rpoBa does not involve a transcription 'juggernaut'}

In $\lambda$ the upstream cis-acting site (nut) serves as a nucleation site for assembly of an antitermination complex or transcription juggernaut (Greenblatt et al., 1993). This termination-resistant elongation complex is capable of transcribing through multiple downstream terminators. Accordingly, one possible explanation for increased readthrough of $r p o B a$ when transcription occurs from weak promoters is that at lower transcription frequencies a higher fraction of transcription complexes become modified during elongation through the $\operatorname{rplJL}$ region in a manner analogous to that of $\lambda$. These modified juggernauts would be resistant to termination and would show increased readthrough at rpoBa. Consequently, this model predicts that the population of transcription complexes which finally emerge downstream of $r p o B a$ should be enriched for modified termination-resistant transcription complexes, and that subsequent readthrough of a second tandem copy of $r p o B a$ should be more efficient than the first.

We tested this by joining an additional copy of a $58 \mathrm{bp}$ DdeI-NarI fragment containing rpoBa onto the end of existing constructs that contained the complete rplJLrpoBa region (HindIII-NarI) in a transcriptional fusion to lacZ. $\beta$-Galactosidase activities of these constructs were then compared with fusions either ending before $r p o B a$ or containing a single copy of $r p o B a$. This allowed us to determine the readthrough of each of the tandem copies of $r p o B a$ (Fig. 4). The results showed that with the strong Pant 285 promoter, readthrough of a single copy of $r p o B a$ in its normal configuration is $23 \%$, but when an additional copy of $r p o B a$ is fused onto the end, readthrough of the second copy drops to $14 \%$. Similarly, with the weaker Pant 224 promoter, transcription across the first copy of $r p o B a$ is $35 \%$, while that of a second copy drops to $22 \%$. Thus, in contrast to the model that some fraction of the transcription complexes traversing the rplrpoBa region become juggernauts, the results showed that the efficiency of 


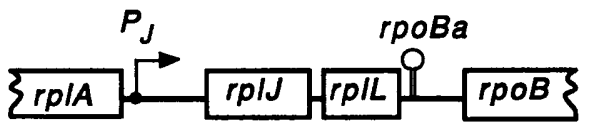

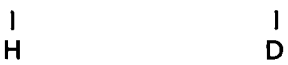

D $\mathrm{N}$

$\beta$-Gal (units)

Total R

(\%)

6388
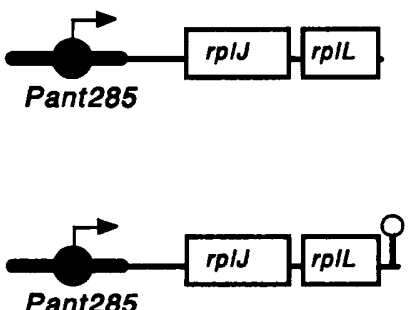

1458

23

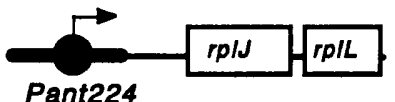

741

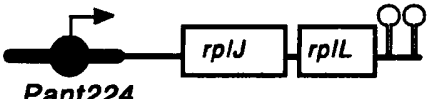

8
RT of 2nd rpoBa (\%)

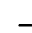

14

Fig. 4. Readthrough of single or tandem copies of rpoBa. Two sets of lacZ transcriptional fusions were constructed with $r p / J p\left(P_{j}\right)$ replaced by either Pant285 or Pant224 attached at the HindIII site located $1379 \mathrm{bp}$ upstream of rpoBa. Each set of fusions contained either no (Ddel, D), one (Narl, N) or two (extra 58 bp Narl-Ddel fragment) copies of rpoBa (represented as a hairpin) at the $3^{\prime}$ end fused to lacZ. Total readthrough frequency (RT) was calculated as the percentage $\beta$-galactosidase activity $(\beta$ gal) for constructs with one or two copies of rpoBa relative to the fusions without $r p o B a$. RT of the second copy of rpoBa was calculated as the percentage $\beta$-gal for constructs with two copies of rpoBa relative to the fusions with one copy. readthrough of the second tandem copy of $r p o B a$ is not increased, in fact somewhat decreased, when transcribed from either the strong or weak promoter.

\section{The cis-acting site upstream of rpoBa does not modulate the efficiency of other terminators}

The nut sites of $\lambda$ also reduce termination when placed upstream of terminators other than the endogenous $\lambda$ terminators. To address the question of whether the region between the SwaI and DdeI sites preceding rpoBa would affect other terminators in the same fashion as $r p o B a$, we placed this $393 \mathrm{bp}$ segment upstream of the $t R 2^{2}$ and $t R 2^{9}$ terminators. Two sets of transcriptional fusions to lac $Z$ were constructed with $t R 2^{2}$, one driven by the strong Pant 285 promoter and the second by the weaker Pant1045. With $t R 2^{9}$ a single set of fusions transcribed by Pant 1045 was assembled. As summarized in Table 3, in no case did the insertion of this region upstream of $t R 2^{2}$ or $t R 2^{9}$ relieve termination. Even when the $393 \mathrm{bp}$ region was placed between a weak promoter and these terminators, conditions that would relieve termination at $r p o B a$, no decrease in termination was observed. This suggests that this site works only in combination with rpoBa to modulate termination.

\section{NusA increases readthrough of rpoBa, but is not involved in transcription-frequency-dependent modulation}

We have previously found evidence, both in vivo (Ralling \& Linn, 1987) and in vitro (Linn \& Greenblatt, 1992), that NusA decreases termination frequency at rpoBa. To test whether NusA played a role in the transcription-frequency-dependent modulation of $r p o B a$, two pairs of $l a c Z$ transcriptional fusions were used to assay $r p o B a$ readthrough in wild-type cells and three nusA mutants, nusA10(Cs) (Schauer et al., 1987), nusA1 (Friedman, 1971) and nusA11(Ts) (Nakamura et al., 1986). One pair of fusions replaced $r p l J p$ with the strong Pant 285 promoter, while the second pair used the weaker Pant1045. The promoters were attached via an engineered HindIII site located $1379 \mathrm{bp}$ upstream of the rpoBa termination site. Since the normal transcription 
Table 3. Effect of $393 \mathrm{bp}$ Swal-Ddel fragment on other terminators

\begin{tabular}{|c|c|c|c|}
\hline Fusion & Features & $\beta$-Gal (units) ${ }^{*}$ & $\mathbf{R T}(\%) \dagger$ \\
\hline $\mathrm{Pa} 285$ & Pant 285 & 7199 & - \\
\hline tR35 & Pant $285+t R 2^{2}$ & 1436 & 20 \\
\hline KS253 & Pant $285+393$ bp $\ddagger$ & 6069 & - \\
\hline $\mathrm{tR} 43$ & Pant $285+393 \mathrm{bp}+t R 2^{2}$ & 1387 & 23 \\
\hline $\mathrm{Pa} 1045$ & Pant1045 & 511 & - \\
\hline tR34 & Pant1045 +tR2 $2^{2}$ & 52 & 10 \\
\hline tR46 & Pant1045 + $393 \mathrm{bp}$ & 545 & - \\
\hline tR40 & Pant $1045+393 \mathrm{bp}+t R 2^{2}$ & 44 & 8 \\
\hline Pa1045 & Pant1045 & 511 & - \\
\hline tR44 & Pant1045+tR2 $2^{9}$ & 161 & 32 \\
\hline $\mathrm{tR} 46$ & Pant1045 + $393 \mathrm{bp}$ & 545 & - \\
\hline $\mathrm{tR} 45$ & Pant1045 $+393 \mathrm{bp}+t R 2^{9}$ & 143 & 27 \\
\hline
\end{tabular}

$* \beta$-Galactosidase activities ( $\beta$-gal) were determined as described in Methods.

$\dagger$ Percentage readthrough frequencies $(\mathrm{RT})$ were calculated as $100 \times(\beta$-gal units for constructs with terminator $\div \beta$-gal units for constructs without terminator).

‡ The 393 bp SwaI-DdeI fragment encompasses the region from $436 \mathrm{bp}$ to $43 \mathrm{bp}$ upstream of the $r p o B a$ termination site.

start site of $r p l J p$ is 8 bp downstream of this HindIII site, these fusions position the promoters so the complete $r p l J$ leader is present. The first construct of each pair contained the complete $r p l J L$ region but ended $43 \mathrm{bp}$ before (DdeI) the rpoBa termination site, while the second construct extended an additional $58 \mathrm{bp}$ to end $15 \mathrm{bp}$ downstream ( $\mathrm{NarI}$ ) of $r p o B a$. $\beta$-Galactosidase activity measurements for the first fusion revealed transcription frequency into the attenuator, whereas measurements for the second fusion revealed readthrough frequency. In nus $A^{+}$cells the frequency of readthrough of $\mathrm{rpoBa}$ was approximately twofold higher with the lower levels of transcription driven by Pant $1045(43 \%)$ as compared to the stronger Pant 285 $(23 \%)$ (Table 4). In the nusA1O(Cs) and nusA1 backgrounds, the level of readthrough decreased modestly, while in the nus $A 11$ (Ts) strain, readthrough decreased to $26 \%$ and $12 \%$, respectively, for the fusions transcribed by the strong and weak promoters. Thus the nusA11(Ts) allele reduced readthrough by approximately half, but none of the nus $A$ mutations significantly altered the twofold difference in readthrough efficiency seen between the high and low levels of transcription.

\section{DISCUSSION}

The efficiency of termination at the $r p o B a$ attenuator was modulated by the frequency with which transcription complexes enter this site. Current models of simple terminator function imply that terminator efficiency should be constant regardless of transcription frequency (Yager \& von Hippel, 1987; von Hippel \& Yager, 1992). We have now directly tested this with four

Table 4. Effect of NusA on termination at rpoBa

\begin{tabular}{|lllrc|}
\hline Fusion & \multicolumn{1}{c}{ Features } & Genotype & $\boldsymbol{\beta}$-Gal (units)† & RT (\%) \\
\hline KS169 & Pant285-rpJL & $n u s A^{+}$ & 6454 & - \\
KS170 & Pant285-rplJLrpoBa & $n u s A^{+}$ & 1468 & 23 \\
KS267 & Pant1045-rplJL & $n u s A^{+}$ & 598 & - \\
KS266 & Pant1045-rplJLrpoBa & $n u s A^{+}$ & 258 & 43 \\
KS169 & Pant285-rplJL & $n u s A 10\langle\mathrm{Cs})$ & 7004 & - \\
KS170 & Pant285-rplJLrpoBa & $n u s A 10(\mathrm{Cs})$ & 1409 & 20 \\
KS267 & Pant1045-rplJL & $n u s A 10(\mathrm{Cs})$ & 737 & - \\
KS266 & Pant1045-rplJLrpoBa & $n u s A 10(\mathrm{Cs})$ & 284 & 37 \\
KS169 & Pant285-rplJL & $n u s A 1$ & 9066 & - \\
KS170 & Pant285-rplJLrpoBa & $n u s A 1$ & 1524 & 17 \\
KS267 & Pant1045-rplJL & $n u s A 1$ & 958 & - \\
KS266 & Pant1045-rplJLrpoBa & $n u s A 1$ & 349 & 36 \\
KS169 & Pant285-rplJL & $n u s A 11(\mathrm{Ts})$ & 10973 & - \\
KS170 & Pant285-rplJLrpoBa & $n u s A 11(\mathrm{Ts})$ & 1321 & 12 \\
KS267 & Pant1045-rplJL & $n u s A 11(\mathrm{Ts})$ & 1406 & - \\
KS266 & Pant1045-rplJLrpoBa & $n u s A 11(\mathrm{Ts})$ & 367 & 26 \\
\hline
\end{tabular}

* The $n u s A^{+}$strain was MG2. The mutated nusA alleles were transferred to MG2 by P1 transduction as described in Methods. Cultures were grown at $37^{\circ} \mathrm{C}$. Additional measurements were conducted with the $n u s A 10(\mathrm{Cs})$ lysogens grown at $33^{\circ} \mathrm{C}$ and the nus $A 1$ and nus $A 11(\mathrm{Ts})$ lysogens grown at $40^{\circ} \mathrm{C}$, but the readthrough frequencies did not change significantly (data not shown).

$\dagger \beta$-Galactosidase activities $(\beta$-gal) were determined as described in Methods.

$\neq$ Percentage readthrough frequencies $(\mathrm{RT})$ were calculated as $100 \times(\beta$-gal units for constructs with terminator $\div \beta$-gal units for constructs without terminator). 
terminators which have termination efficiencies of approximately $98-68 \%$. All of these terminators had fairly constant termination efficiencies over a wide range of transcription frequency. Since the efficiency of $t R 2^{9}$ in particular is in the same range as that of $r p o B a$, it argues that a decrease in termination efficiency with decreasing transcription frequency is not an inherent property of weaker terminators and may be unique to $r p o B a$.

In the lytic cycle of $\lambda$, the phage-encoded $\mathrm{N}$ protein acts with four $E$. coli proteins, NusA, NusB, NusG and S10, to modify RNA polymerase such that it fails to respond to termination signals. This modification requires transcription through a cis-acting site $(n u t)$ located upstream of the terminators. The nascent nut RNA is an essential component in the ribonucleoprotein complex that assembles on the surface of the transcribing RNA polymerase. This modified complex, which contains $\mathrm{N}$, NusA, NusB, NusG, S10 and nut RNA, becomes a transcriptional juggernaut with an enhanced ability to processively read through multiple terminators (Greenblatt et al., 1993). Since termination efficiency at $r p o B a$ can be modulated and because our previous results indicated that both NusA and NusG appear to play a role in termination at this site, we tested whether the mechanism operating at $r p o B a$ was analogous to the $\lambda$ antitermination system.

Analysis of deletions upstream of $r p o B a$ suggested that at least part of the sequences required for discriminating between high and low frequency transcription, and adjusting $r p o B a$ readthrough accordingly, may map within the $165 \mathrm{bp}$ interval between the Swal site at the start of $r p l L$ and the EcoRI site within rplL. The observation that two different upstream endpoints (HindIII and SwaI) show significant differences in $r p o B a$ readthrough for high and low transcription frequency, while two other endpoints $(E c o \mathrm{RI}$ and $A l u \mathrm{I})$ no longer show this difference, argues that the apparent loss of $r p o B a$ modulation is not likely to be due to a novel fusion junction between the Pant and rplJL sequences that interferes in some way with modulation.

The deletion analysis did not distinguish whether the functional form of this putative region was RNA or DNA. In N-mediated antitermination it is the nut site RNA that functions as part of the termination resistant elongation complex. The Q-mediated antitermination system of $\lambda$, which is responsible for expression of the late genes, also requires a cis-acting site $(q u t)$, but this site functions in the form of DNA. Shortly after initiation, the polymerase pauses at the downstream section of the qut site, but when $\mathrm{Q}$, in the presence of NusA, interacts with the upstream sequence of qut, polymerase is accelerated out of this site and is transformed into a termination-resistant complex (Yarnell \& Roberts, 1992; Greenblatt et al., 1993). Further work will be required to more precisely map the site upstream of $r p o B a$ and determine if it functions as DNA or RNA.

The nut sites of $\lambda$ can also decrease termination at heterologous terminators when placed upstream. How- ever when the $393 \mathrm{bp} S w a \mathrm{I}-D d e \mathrm{I}$ fragment upstream of rpoBa was inserted in front of the $t R 2^{2}$ and $t R 2^{9}$ terminators there was no reduction in termination even at low levels of transcription. This suggests that the cisacting site found in this region works only in combination with $r p o B a$ to modulate termination in response to transcription frequency and is not a general antitermination site as are the nut sites. Also, the result that a second copy of $r p o B a$ placed downstream of the original had a termination efficiency no lower than the first copy indicates those transcription complexes that read through $r p o B a$ do not appear to be a select class of termination-resistant complexes. This result does not rule out the possibility that RNA polymerase passing through the SwaI-EcoRI region becomes modified in some fashion that reduces termination at $r p o B a$. It simply argues that this putative modification only enhances readthrough at the first copy of $r p o B a$ and is not a persistent modification that allows the processive antitermination seen with the $\mathrm{N}$-modified transcriptional juggernauts. If polymerase was to pause at the cisacting site upstream of $r p o B a$ and supposing some step in the modification was rate-limiting, then a higher fraction of transcription complexes may become modified at lower transcription frequencies. This could explain the increased percentage of polymerase molecules seen reading through $r p o B a$ at lower transcription frequencies.

If NusA was essential for regulating the transcriptionfrequency-dependent efficiency of $r p o B a$ then in a nus $A$ mutant the difference in readthrough at high and low levels of transcription should have disappeared. The nusA10(Cs) and nusA1 mutants showed only a modest effect, while in the nusA11(Ts) mutant readthrough of $r p o B a$ decreased to approximately half that seen in the wild-type strain, confirming that NusA normally increases readthrough at this site. However, in the mutants the ratio of readthrough at high and low levels of transcription did not vary significantly from the wildtype strain, arguing that NusA is not directly involved in transcription-frequency-dependent modulation. The nusA11(Ts) mutation changes a single nucleotide at position 542 of the coding sequence, while nus $A 1$ has a single change at nucleotide 548 and $n u s A 10(\mathrm{Cs})$ has two changes at nucleotides 311 and 634 (Craven \& Friedman, 1991). Each of these alterations causes a single amino acid change in the protein. The fact that nusA11(Ts) has a greater effect at $r p o B a$ suggests that the amino acid residue altered by this mutation is directly involved in the interactions that decrease termination at rpoBa. Alternatively, this change may have a greater detrimental effect on the overall structure or stability of NusA under the conditions tested.

The regulation of termination efficiency at $r p o B a$ has some parallels with the $\mathrm{N}$ and $\mathrm{Q}$ antitermination systems of $\lambda$ in that NusA increases readthrough and also there appears to be the involvement of a cis-acting upstream element. However, in contrast to $\lambda$, the upstream element functions only in combination with $r p o B a$, the 
polymerase molecules traversing this region do not become transcriptional juggernauts and NusA does not appear to be essential for transcription-frequencydependent modulation. The apparent terminator specificity of the upstream site and the lack of processivity might suggest a direct mRNA antiterminatorterminator interaction. Such a mechanism would be similar in principle to that seen in the $\operatorname{trp}$ operon and other amino acid biosynthetic operons (Landick \& Yanofsky, 1987), but here the antiterminatorterminator interaction would be long-range, with more than 270 nucleotides separating these sites. However, sequence analysis has not shown any compelling complementarity between $r p o B a$ and the upstream region. Moreover, since the 165 bp Swal-EcoRI interval lies within the rplL gene, one would expect that translation of this ribosomal protein sequence would inhibit formation of a long-range mRNA secondary structure. Also, it is not obvious how such an antiterminatorterminator interaction would be responsive to transcription levels. The transcription frequency, and thus the spacing of the RNA polymerase molecules elongating through this region, might be expected to affect the transient local superhelicity, which in turn may play some role in modulating $r p o B a$ function. Additional studies will be required to further elucidate the mechanism that senses the frequency with which transcription complexes approach $r p o B a$ and then adjusts termination efficiency accordingly.

\section{ACKNOWLEDGEMENTS}

This work was supported by the Canadian Medical Research Council. We thank D. Friedman for supplying the nusA mutant strains K1457, K1914 and K3903, and also the $t R 2^{2}$ and $t R 2^{9}$ terminators. We thank M. Susskind for supplying the P22 ant promoter mutations.

\section{REFERENCES}

Appleyard, R. K. (1954). Segregation of new lysogenic types during growth of a doubly lysogenic strain derived from Escherichia coli K12. Genetics 39, 440-452.

Brosius, J., Dull, T. J., Sleater, D. D. \& Noller, H. F. (1981). Gene organization and primary structure of a ribosomal RNA operon from Escherichia coli. J Mol Biol 148, 107-127.

Cheng, S. C., Lynch, E. C., Leason, K. R., Court, D. L., Shapiro, B. A. \& Friedman, D. J. (1991). Functional importance of sequence in the stem-loop of a transcriptional terminator. Science 254, $1205-1207$.

Clark, D. J. \& Maaloe, O. (1967). DNA replication and the division cycle in Escherichia coli. J Mol Biol 23, 99-112.

Craven, M. G. \& Friedman, D. I. (1991). Analysis of the Escherichia coli nus $A 10(\mathrm{Cs})$ allele: relating nucleotide changes to phenotypes. J Bacteriol 173, 1485-1491.

Das, A. (1992). How the phage lambda $N$ gene product suppresses transcription termination: communication of RNA polymerase with regulatory proteins mediated by signals in nascent RNA. J Bacteriol 174, 6711-6716.

Dykxhoorn, D. M., St Pierre, R. \& Linn, T. (1996). Synthesis of the $\beta$ and $\beta^{\prime}$ subunits of Escherichia coli RNA polymerase is autogeneously regulated in vivo by both transcriptional and translational mechanisms. Mol Microbiol 19, 483-493.

Engbaek, F., Gross, C. \& Burgess, R. R. (1976). Quantitation of RNA polymerase subunits in Escherichia coli during exponential growth and after bacteriophage T4 infection. Mol Gen Genet 143, 291-295.

Friedman, D. I. (1971). A bacterial mutant affecting lambda development. In The Bacteriophage Lambda, pp. 733-738. Edited by D. Hershey. Cold Spring Harbor, NY: Cold Spring Harbor Laboratory.

Friedman, D. I. (1988). Regulation of gene expression by termination and antitermination of transcription. In The Bacteriophages, vol. 2, pp. 263-319. Edited by R. Calendar. New York: Plenum.

Greenblatt, J., Nodwell, J. R. \& Mason, S. W. (1993). Transcriptional antitermination. Nature 364, 401-406.

Hanahan, D. (1985). Techniques for transformation of E. coli. In DNA Cloning, vol. 1, pp. 109-135. Edited by D. M. Glover. Oxford: IRL Press.

Hayward, R. S. \& Fyfe, S. K. (1978). Oversynthesis and instability of sigma protein in a merodiploid strain of Escherichia coli. Mol Gen Genet 159, 89-99.

von Hippel, P. H. \& Yager, T. D. (1992). The elongation-termination decision in transcription. Science 255, 809-812.

Holmes, D. S. \& Quigley, M. (1981). A rapid boiling method for the preparation of bacterial plasmids. Anal Biochem 114, 193-197.

Ishihama, A. (1993). Protein-protein communication within the transcription apparatus. J Bacteriol 175, 2483-2489.

Iwakura, Y., Ito, K. \& Ishihama, A. (1974). Biosynthesis of RNA polymerase in Escherichia coli. I. Control of RNA polymerase content at various growth rates. Mol Gen Genet 133, 1-23.

Kawakami, K., Saitoh, T. \& Ishihama, A. (1979). Biosynthesis of RNA polymerase in Escherichia coli. IX. Growth-dependent variations in the synthesis rate, content and distribution of RNA polymerase. Mol Gen Genet 174, 107-116.

Landick, R. \& Yanofsky, C. (1987). Transcription attenuation. In Escherichia coli and Salmonella typhimurium: Cellular and Molecular Biology, pp. 1276-1301. Edited by F. C. Neidhardt and others. Washington, DC: American Society for Microbiology.

Linn, T. \& Greenblatt, J. (1992). The NusA and NusG proteins of Escherichia coli increase the in vitro readthrough frequency of a transcriptional attenuator preceding the gene for the $\beta$ subunit of RNA polymerase. J Biol Chem 267, 1449-1454.

Linn, T. \& St Pierre, R. (1990). Improved vector system for constructing transcriptional fusions that ensures independent translation of lacZ. J Bacteriol 172, 1077-1084.

Maniatis, T., Fritsch, E. F. \& Sambrook, J. (1982). Molecular Cloning: a Laboratory Manual. Cold Spring Harbor, NY: Cold Spring Harbor Laboratory.

Miller, J. H. (1972). Experiments in Molecular Genetics. Cold Spring Harbor, NY: Cold Spring Harbor Laboratory.

Mousset, S. \& Thomas, R. (1969). Ter, a function which generates the ends of the mature lambda chromosome. Nature 221, 242-244.

Moyle, H., Waldburger, C. \& Susskind, M. M. (1991). Hierarchies of base pair preferences in the p22 ant promoter. J Bacteriol 173, 1944-1950.

Nakamura, Y., Mizusawa, S., Court, D. L. \& Tsugawa, A. (1986). Regulatory defects of a conditionally lethal nusAts mutant of Escherichia coli. Positive and negative modulator roles of NusA protein in vivo. J Mol Biol 189, 103-111. 
Pedersen, S., Reeh, S. V., Parker, J., Watson, R. J., Friesen, J. D. \& Fiil, N. P. (1976). Analysis of the proteins synthesized in ultraviolet light-irradiated Escherichia coli following infection with the bacteriophage $\lambda \mathrm{drif}^{\mathrm{d}} 18$ and $\lambda \mathrm{dfus} 3$. Mol Gen Genet 144, 339-343.

Post, L. E., Strycharz, G. D., Nomura, M., Lewis, H. \& Dennis, P. P. (1979). Nucleotide sequence of the ribosomal protein gene cluster adjacent to the gene for RNA polymerase subunit beta in Escherichia coli. Proc Natl Acad Sci USA 76, 1697-1701.

Ralling, G. \& Linn, T. (1987). Evidence that Rho and NusA are involved in termination in the $r p l L-r p o B$ intercistronic region. $J$ Bacteriol 169, 2277-2280.

Ralling, G., Bodrug, S. \& Linn, T. (1985). Growth rate-dependent regulation of RNA polymerase synthesis in Escherichia coli. Mol Gen Genet 201, 379-386.

Sambrook, J., Fritsch, E. F. \& Maniatis, T. (1989). Molecular Cloning: a Laboratory Manual, 2nd edn. Cold Spring Harbor, NY: Cold Spring Harbor Laboratory.

Santos, M. A. (1991). An improved method for the small scale preparation of bacteriophage DNA based on phage precipitation by zinc chloride. Nucleic Acids Res 19, 5442.

Schauer, A. T., Carver, D. L., Bigelow, B., Baron, L. S. \& Friedman, D. I. (1987). Lambda $\mathrm{N}$ antitermination system: functional analysis of phage interactions with the host NusA protein. $J \mathrm{Mol}$ Biol 194, 679-690.

Schmidt, M. C. \& Chamberlin, M. J. (1987). NusA protein of Escherichia coli is an efficient transcription termination factor for certain terminator sites. $J$ Mol Biol 195, 809-818.

Steward, K. L. \& Linn, T. (1991). In vivo analysis of overlapping transcription units in the $r p l K A J L r p o B C$ ribosomal protein-RNA polymerase gene cluster of Escherichia coli. J Mol Biol 218, 23-31.

Steward, K. \& Linn, T. (1992). Transcription frequency modulates the efficiency of an attenuator preceding the $r p o B C$ RNA polymerase genes of Escherichia coli: possible autogenous control. Nucleic Acids Res 20, 4773-4779.

Yager, T. D. \& von Hippel, P. H. (1987). Transcript elongation and termination in Escherichia coli. In Escherichia coli and Salmonella typhimurium: Cellular and Molecular Biology, pp. 1241-1275. Edited by F. C. Neidhardt and others. Washington, DC: American Society for Microbiology.

Yarnell, W. S. \& Roberts, J. W. (1992). The phage $\lambda$ gene $Q$ transcription antiterminator binds DNA in the late gene promoter as it modifies RNA polymerase. Cell 69, 1181-1189.

Received 1 April 1997; revised 6 July 1997; accepted 11 July 1997. 